\title{
ORIENTATION PRESERVING ACTIONS OF FINITE ABELIAN GROUPS ON SPHERES
}

\author{
RONALD M. DOTZEL
}

\begin{abstract}
If $G$ is a finite Abelian group acting as a $\mathbf{Z}_{(\mathcal{P})}$-homology $n$-sphere $X$ (where $P$ is the set of primes dividing $|G|$ ), then there is an integer valued function $n(, G)$ defined on the prime power subgroups $H$ of $G$ such that $X^{H}$ has the $\mathbf{Z}_{(p)}$-homology of a sphere $S^{n(H, G)}$. We prove here that there exists a real representation $R$ of $G$ such that for any prime power subgroup $H$ of $G$, $\operatorname{dim}\left(S\left(R^{H}\right)\right)=n(H, G)$ where $S\left(R^{H}\right)$ is the unit sphere of $R^{H}$, provided that $n-n(H, G)$ is even whenever $H$ is a 2-subgroup of $G$.
\end{abstract}

0. Introduction. Suppose that $G$ is a finite Abelian group and let $P$ be the set of primes dividing $|G|$. If $G$ acts on a finite $C W$-complex $X$ which has the $\mathbf{Z}_{(P)}$ homology of $S^{n}$, then for any $p \in \mathcal{P}$ and $p$-subgroup $H$ of $G$ the fixed point set of $H$ on $X, X^{H}$, has the $\mathbf{Z}_{(p)}$-homology of $S^{n(H, G)}$ for some integer $n(H, G) \geq-1$ $(-1$ signifies empty). This is a well-known consequence of Smith theory ([2, III or 1, IV], e.g.). Thus we obtain in this way an integer valued function, $n(, G)$, defined on the set of $p$-subgroups of $G$ by $H \mapsto n(H, G)$ (note that $n(e, G)=n$ ). This function is called the "dimension function" and has a considerable literature (see $[\mathbf{5}, \mathbf{3}, \mathbf{6}, \mathbf{7}, \mathbf{9}] ;[\mathbf{1 0}]$ gives a related extensive bibliography).

The function $n(, G)$ satisfies the following well-known conditions (see [1, XIII, $2.3 ; \mathrm{IV}, 4.4,4.7])$ :

1. (Borel Formula) If $H \leq K$ are both $p$-subgroups of $G$ and $K / H=\mathbf{Z}_{p}+\mathbf{Z}_{p}$, then $n(H, G)-n(K, G)=\sum\left(n\left(K^{\prime}, G\right)-n(K, G)\right)$ with the sum over all $H \leq K^{\prime} \leq$ $K$ such that $K^{\prime} / H=\mathbf{Z}_{p}$.

2. If $H \leq K$ are $p$-subgroups of $G$, then $n(K, G) \leq n(H, G)$.

3. If $H \leq K$ are $p$-subgroups of $G$ with $K / H=\mathbf{Z}_{p}$ and $p$ odd, then $n(H, G)-$ $n(K, G)$ is even.

4. If $H \leq K^{\prime} \leq K$ are 2-subgroups of $G$ such that $K / H=\mathbf{Z}_{4}, K^{\prime} / H=\mathbf{Z}_{2}$, then $n(H, G)-n\left(K^{\prime}, G\right)$ is even.

For each $p \in \mathcal{P}$, let $G(p)$ denote the $p$-Sylow subgroup of $G$ and set $N(, G)=$ $n(, G)+1$. The function $N(, G)$ restricted to the subgroups of $G(p)$ will naturally be denoted by $N(, G(p))$. In [8] it was shown that $N(, G(p))$ is realized by a real representation $V(p)$ of $G(p)$ which means that for each $H \leq G(p)$, $\operatorname{dim} V(p)^{H}=N(H, G(p))=N(H, G)$. If $S(V(p))$ denotes the unit sphere of $V(p)$, then $\operatorname{dim} S\left(V(p)^{H}\right)=n(H, G)$.

Here we are interested in the existence of a real representation $R$ of the Abelian group $G$ such that for any $p$-subgroup $H$ of $G$ (for any $p \in \mathcal{P}$ ), $\operatorname{dim} R^{H}=N(H, G)$. Thus $R$ would be a simultaneous realization of the functions $N(, G(p)), p \in P$.

Received by the editors March 22, 1985. These results were presented to the AMS at the 829 th meeting in Charlotte, North Carolina, in October 1986.

1980 Mathematics Subject Classification (1985 Revision). Primary 57S17, 57S25. 
It should be noted that in the special case where $X^{H}$ is a homology sphere for all $H \leq G$, then $N(, G)$ is defined on all subgroups of $G$ and tom Dieck has shown in $[3]$ that $N(, G)$ is realized by a difference of representations. In general, this is best possible.

Clearly $N(, G)$ satisfies conditions $1-4$ if $n(, G)$ does. We will denote $N(e, G)$ by $N$. As an example to show that some condition beyond 1-4 is needed, suppose $G=$ $\mathbf{Z}_{6}, N=2, N\left(\mathbf{Z}_{3}, G\right)=0$, and $N\left(\mathbf{Z}_{2}, G\right)=1$. Then $N(, G)$ satisfies conditions 1-4 but there is no real representation of $G$ which realizes these numbers simultaneously as dimensions. From now on we will assume the following "orientation preserving" condition holds, in addition to conditions 1-4:

5. If $H$ is any 2-subgroup of $G$, then $N-N(H, G)$ is even.

We obtain the following theorem and corollary:

THEOREM. Let $G$ be a finite Abelian group and suppose $N(, G)$ is a nonnegative integer valued function defined on the p-subgroups of $G$ for all $p|| G \mid$, satisfying conditions 1-5. Then there exists a real representation $R$ of $G$ such that for any p-subgroup $H$ of $G, p|| G \mid, \operatorname{dim} R^{H}=N(H, G)$. Furthermore, if $\bar{R}$ is another such representation of $G$ then for all subgroups $H$ of $G$,

$$
\operatorname{dim} R^{H} \equiv \operatorname{dim} \bar{R}^{H} \quad(\bmod 2) .
$$

COROLlaRY. Let $G$ be a finite Abelian group and suppose the 2-Sylow subgroup of $G$ is cyclic. If $N(, G)$ is a nonnegative integer valued function defined on the p-subgroups of $G, p|| G \mid$ satisfying only conditions $1-4$, then there exists a real representation $R$ such that for any p-subgroup $H$ of $G, p|| G \mid, \operatorname{dim} R^{H}=N(H, G)+$ 1.

In $\S \S 1$ and 2 we prove the theorem and corollary respectively. We thank the referee for several suggestions leading to an improved exposition.

1. Proof of the theorem. Let $G$ be a finite Abelian group and suppose $N(, G)$ is a nonnegative integer valued function on the $p$-subgroups of $G$, for all $p \in P$, satisfying conditions $1-5$. By [8], for each $p \in P$ there is a representation $V(p)$ of the $p$-Sylow subgroup $G(p)$ of $G$ such that $\operatorname{dim} V(p)^{H}=N(H, G(p))$ for all $H \leq G(p)$. Let $\mathcal{V}=\bigotimes_{p|| G \mid} V(p)$. Then $\mathcal{V}$ is a representation of $G$ and we will prove by induction on $|G|$ that $\mathcal{V}$ contains a subrepresentation $R$ of $G$ of dimension $N=N(e, G)$ such that $\operatorname{dim} R^{H}=N(H, G)$ for all $p$-subgroups $H$ of $G$, all $p \in P$. So if $|K|<|G|, N(, K)$ is a nonnegative integer valued function on the prime power subgroups of $K$ and $W(p)$ is a representation of $K(p)$ realizing $N(, K(p))$, we can assume $\mathcal{W}=\bigotimes_{p|| K \mid} W(p)$ contains a subrepresentation realizing $N(, K)$.

Suppose that $N(, G)$ is a nonnegative integer valued function defined on the prime power order subgroups of an Abelian group $G$ satisfying conditions 1-5 and suppose $N(e, G)=N(H, G)$ for some $H \leq G(p),|H|=p$. Then for any prime power order subgroup $K$ of $G$, define $N(K / K \cap H, G / H)=N(K, G)$. It is clear that $N(, G / H)$ satisfies conditions 1-5. Moreover, for any $K \leq G(p)$,

$$
N(K, G) \stackrel{\text { def }}{=} N(K, G(p))=N(K H, G(p)) \stackrel{\text { def }}{=} N(K H, G) \stackrel{\text { def }}{=} N(K H / H, G / H) .
$$

For by induction we can assume $N\left(K^{\prime}, G(p)\right)=N\left(K^{\prime} H, G(p)\right)$ for any $K^{\prime} \$ K$ and clearly we can assume $H \not K$. Select $K^{\prime}<K$ such that $\left|K / K^{\prime}\right|=p$ and use 
condition 1 (Borel Formula) on $K^{\prime} \leq K H$ to obtain $N(K, G(p))=N(K H, G(p))$. It follows that in this case, a representation of $G / H$ realizing $N(, G / H)$ can be regarded a s an unfaithful representation of $G$ (with kernel at least $H$ ) realizing $N(, G)$.

Now for each $p \in \mathcal{P}$ and each $\mathbf{Z}_{p} \leq G$, we must have $N-N\left(\mathbf{Z}_{p}, G\right)>0$, otherwise by the observation above we could assume we are given a dimension function on $G / \mathbf{Z}_{p}$. Of all the differences $N-N\left(\mathbf{Z}_{p}, G\right), p \in \mathcal{P}$, let $p_{0}$ and $H_{0}=\mathbf{Z}_{p_{0}}$ be such that $N-N\left(H_{0}, G\right)$ is a minimum. Then the representation $V\left(p_{0}\right)$ of $G\left(p_{0}\right)$ (the $p_{0}$-Sylow subgroup of $G$ ) contains an irreducible subrepresentation $W\left(p_{0}\right)$ of $G\left(p_{0}\right)$ on which $H_{0}$ acts without (nonzero) fixed points. For $q \neq p_{0}$ select $H=\mathbf{Z}_{q} \leq G(q)$ such that $N-N(H, G)$ is least for $q$ (in general $N-N\left(H_{0}, G\right) \leq N-N(H, G)$ ) and let $W(q)$ be an irreducible subrepresentation of $V(q)$ on which $H$ acts without fixed points. Then $\mathcal{W}=\bigotimes_{q|| G \mid} W(q)$ is a $G$-subrepresentation of $\mathcal{V}=\bigotimes_{q|| G \mid} V(q)$. Let $R_{1}$ be an irreducible $G$-subrepresentation of $\mathcal{W}$. If $|G|$ is larger than $2, R_{1}$ has dimension 2 , since $R_{1}$ induces a free, irreducible, real representation of a cyclic group of order larger than 2 (the cyclic group is $G /$ kernel of $R_{1}=$ kernel of $\mathcal{W}$ ).

Now $R_{1}$, is being a representation of $G$, has associated to it a dimension function $N_{1}(, G)$ defined on all subgroups $H$ of $G$ by $N_{1}(H, G)=\operatorname{dim} R_{1}^{H}$. Set $\bar{N}_{1}(, G)=$ $N(, G)-N_{1}(, G)$. It is easy to verify that $\bar{N}_{1}(, G)$ is a dimension function defined on the prime power subgroups of $G$ satisfying conditions 1-5.

Since $\bar{N}_{1}(e, G)<N(e, G)$ and $\bar{N}_{1}\left(H_{0}, G\right)=N\left(H_{0}, G\right)$ we are presented with two situations: (a) $\bar{N}_{1}(e, G)=\bar{N}_{1}\left(H_{0}, G\right)$ or (b) $\bar{N}_{1}(e, G)>\bar{N}_{1}\left(H_{0}, G\right)$.

In case (a) the function $\bar{N}_{1}(, G)$ may be replaced (as noted above) by a dimension function defined on the prime power subgroups of $G / H_{0}$. Since for any $q$-subgroup $K$ of $G, \operatorname{dim} R_{1}^{K}=\operatorname{dim} W(q)^{K}$, the subrepresentation $W(q)^{\perp}$ of $V(q)$ realizes the dimension function $\bar{N}_{1}(, G(q))$. Since $\left|G / H_{0}\right|<|G|$ by induction the tensor product of all the $W(q)^{\perp}$ contains a subrepresentation $R^{*}$ of $G / H_{0}$ (which may be thought of as an unfaithful representation of $G$ ). $R^{*}$ is a $G$-subrepresentation of $\mathcal{V}$, the tensor product of all the $V(q) . R^{*} \oplus R_{1}$ is the required representation in this case.

In (b), where we have $\bar{N}_{1}(e, G)>\bar{N}_{1}\left(H_{0}, G\right)$, note that $\bar{N}_{1}(e, G)-\bar{N}_{1}\left(H_{0}, G\right)$ is still a minimum of all differences $\bar{N}_{1}(e, G)-\bar{N}_{1}(H, G)$. Since the function $\bar{N}_{1}(, G(Q))$ is realized by the subrepresentation $W(q)^{\perp}$ of $V(q)$, we can repeat the procedure again obtaining another irreducible subrepresentation $R_{2}$ or $G$ with an associated dimension function $N_{2}(, G)$ defined on the prime power subgroups of $G$ (it is the restriction of a dimension function defined on all subgroups of $G$ ). Letting $\bar{N}_{2}(, G)=\bar{N}_{1}(, G)=N_{2}(, G)$ we again have a dimension function satisfying conditions 1-5 and we proceed as above. Eventually we obtain a dimension function $\bar{N}_{k}(, G)$ such that $\bar{N}_{k}(e, G)=\bar{N}_{k}\left(H_{0}, G\right)\left(k=N-N\left(H_{0}, G\right)\right)$. By case (a) and induction there is a $G$-subrepresentation of $\mathcal{V}, R^{*}$ realizing $\bar{N}_{k}(, G)$. The representation $R=R^{*} \oplus R_{1} \oplus R_{2} \oplus \cdots \oplus R_{k}$ is the required $G$-subrepresentation of $v$.

Now suppose $\bar{R}$ is another $G$-subrepresentation such that for any prime power order subgroup $H$ of $G, \operatorname{dim} \bar{R}^{H}=N(H, G)$. Let $H$ be an arbitrary subgroup of $G$ and by induction assume $\operatorname{dim} \bar{R}^{K}-\operatorname{dim} R^{k}$ is even for all subgroups $K$ of $G$ with $|K|<|H|$. Select $K \leq H$ so that $|H / K|$ is an odd prime $p$ (if this is not possible 
then $H$ is a 2-group and $\operatorname{dim} \bar{R}^{H}-\operatorname{dim} R^{H}$ is zero). The group $H / K=\mathbf{Z}_{p}$ acts on both $\bar{R}^{K}$ and $R^{K}$. It follows that both $\operatorname{dim} \bar{R}^{K}-\operatorname{dim} \bar{R}^{H}$ and $\operatorname{dim} R^{K}-\operatorname{dim} R^{H}$ are even and therefore $\operatorname{dim} \bar{R}^{H}-\operatorname{dim} R^{H}$ is even. This completes the proof of the theorem.

2. Proof of the corollary. Suppose $G$ is a finite Abelian group with cyclic 2Sylow subgroup, $G(2)$, and suppose $N(, G)$ is a nonnegative integer valued function defined on the $p$-subgroups of $G, p|| G \mid$, satisfying conditions 1-4. By condition 4, for any proper subgroup $H$ of $G(2), N-N(H, G)$ is even. Suppose that $N-$ $N(G(2), G)$ is odd. For each $p|| G \mid$, let $\bar{V}(p)=V(p) \oplus 1$, where 1 denotes the trivial one-dimensional representation of $G(p)$. It is easy to see that $\bar{V}(p)$ realizes $N(, G(p))+1$. The function $N^{*}(, G)=N(, G)+1$ corresponds to the $G$-action on the unreduced suspension on $X$.

Now since $N-N(G(2), G)$ is odd, $\bar{V}(2)$ has an irreducible summand of dimension 1 on which $H$, the maximal proper subgroup of $G(2)$, acts trivially and on which $G(2)$ acts nontrivially. Denote this summand by $W(2)$ for any $p \neq 2$ let $W(p)$ be a one-dimensinal trivial subrepresentation of $\bar{V}(p)$. Then $R_{1} \bigotimes_{p|| G \mid} W(p)$ is a 1-dimensional $G$-representation with a very large kernel and is a subrepresentation of $\bar{V}=\bigotimes_{p|| G \mid} \bar{V}(p)$. Let $N_{1}(, G)$ be the dimension function associated with $R_{1}$ $\left(N_{1}(, G)\right.$ is actually defined on all subgroups of $\left.G\right)$. Setting $\bar{N}(, G)=N^{*}(, G)-$ $N_{1}(, G)$ we see that $N-\bar{N}(H, G)$ is now even for all prime power subgroups of $G$ so $\bar{N}(, G)$ satisfies conditions $1-5$. By the argument $\S 1, \bar{N}(, G)$ is realized by a subrepresentation $R$ of $\bigotimes_{p|| G \mid} W(P)^{\perp}$, since $\bar{N}(, G(p))$ is realized by $W(p)^{\perp}$ for all $p|| G \mid$. Then $R \oplus R_{1}$ is a subrepresentation of the $G$-representation $\bar{V}$ which realizes $N^{*}(, G)$. This establishes the corollary.

EXAMPLES. Let $G=\mathbf{Z}_{6}, N=2, N\left(\mathbf{Z}_{2}, G\right)=1, N\left(\mathbf{Z}_{3}, G\right)=0$. If we "suspend" $N(, G)$ we have $N^{*}=3, N^{*}\left(\mathbf{Z}_{2}, G\right)=2, N^{*}\left(\mathbf{Z}_{3}, G\right)=1$. Then the construction of $\S \S 2$ and 1 yeilds the 3 -dimensional representation of $G$, given on a generator by

$$
\left(\begin{array}{ccr}
-1 & 0 & 0 \\
0 & R(2 \pi / 3) \\
0 & &
\end{array}\right)
$$

where $R(2 \pi / 3)$ is a $2 \times 2$ rotation matrix.

\section{REFERENCES}

1. A. Borel et al., Seminar on transformation groups, Ann. of Math. Studies, no. 46, Princeton Univ. Press, Princeton, N. J.,

2. G. Bredon, Introduction to compact transformation groups, Academic Press, New York,

3. T. tom Dieck, Homotopiedarstellungen endlicher Gruppen: Dimensionsfunktionen, Invent. Math. 67 (1982), 231-252.

4. __ Transformation groups and representation theory, Lecture Notes in Math., vol. 766, Springer-Verlag, 1979.

5. __ Semi-linear group actions on spheres: Dimension functions, Lecture Notes in Math., vol. 763, Springer-Verlag, 1978, pp. 448-457.

6. T. tom Dieck and T. Petrie, Homotopy rerpesentation of finite groups, Inst. Hautes Étude Sci. Publ. Math. 56 (1983), 129-169.

7. __ Geometric modules over the Burnside ring, Invent. Math. 47 (1978), 273-287. 
8. R. Dotzel and G. Hamrick, p-group actions on homology spheres, Invent. Math. 62 (1981), 437442.

9. K. H. Dovermann and T. Petrie, Artin relations for smooth representations, Proc. Nat. Acad. Sci. U.S.A. 77 (1980), 5620-5621.

10. R. Schultz, Nonlinear analogs of linear group actions, Bull. Amer. Math. Soc. (N.S.) 11 (1984), 263-285.

Department of Mathematical Sciences, University of Missouri-St. Louis, ST. LOUIS, MISSOURI 63121 